\title{
An Interference-Resilient Body Channel Transceiver for Wearable Body Sensor Network
}

\author{
Namjun Cho, Joonsung Bae, and Hoi-Jun Yoo \\ Dept. of EECS, Korea Advanced Institute of Science and Technology (KAIST) \\ 373-1, Guseong-dong, Yuseong-gu, Daejeon, 305-701, Republic of Korea \\ E-mail : buzm@eeinfo.kaist.ac.kr
}

\begin{abstract}
This work deals with a reliability issue of the body channel communication which uses the human body as a signal transmission medium. Due to the body antenna effect, lots of electromagnetic signals are coupled to the on-body receiver, degrading the SIR significantly. In order to reject the in-band interferences, an adaptive frequency hopping scheme using variable sub-channels is proposed. The use of dual frequency synthesizers for frequency hopping improves the data throughput and the acquisition time of the transceiver by eliminating the blank time overhead. The AFH transceiver realized with $0.18 \mu \mathrm{m}$ CMOS process sustains $1.25 \mathrm{Mb} / \mathrm{s}$ throughput when the 10 interferences causing -25dB SIR exist.
\end{abstract}

\section{INTRODUCTION}

Body channel communication (BCC) of using the human body as a signal transmission medium has many advantages for the wearable body sensor network. Since the communication channel is formed by attaching a metal electrode to the human skin [1], most of the wearable health monitoring devices including ECG, EMG, and bodytemperature sensors can transmit data directly through their sensing electrodes. The use of the lower carrier frequency without a large antenna also reduces the power consumption and size of the on-body transceiver efficiently [2].

Since the BCC was first introduced by Zimmermann [3], most studies have concentrated on increasing the data rate and reducing the size of the transceiver $[2,4]$. However, the reliability issues of the $\mathrm{BCC}$ are not addressed properly. In this paper, the body antenna effect, that is the human bodyincurred electromagnetic coupling between the body channel receiver and the adjacent wireless devices, is described. To reject the body antenna effect efficiently, an adaptive frequency hopping $(\mathrm{AFH})$ scheme using the variable number of sub-channels is proposed. Through the chip implementation of the $\mathrm{AFH}$ transceiver, $25 \mathrm{~dB}$ rejection of the interferences is observed, maintaining the data throughput larger than $1.25 \mathrm{Mb} / \mathrm{s}$.

\section{BODY ANTENNA EFFECT}

Fig. 1 shows that the human body under electromagnetic fields behaves as an antenna with its resonance frequency determined by the wavelength $(\lambda)$ equal to twice of the human height. In case that the body is grounded, the resonance wavelength $(\lambda)$ is equal to four times of the height due to the mirror effect [5]. This means that the body resonance may occur at different frequencies, depending on the environmental conditions. Since the human body is a lossy conductor with its conductance value of $<0.1 \mathrm{~S} / \mathrm{m}$ and has a complex shape, the resonance peaking is not sharp but broadly distributed. Consequently, the human body operates as a wide-band antenna in $30-400 \mathrm{MHz}$ frequency range, which overlaps the optimum $10-120 \mathrm{MHz}$ band for BCC in terms of the SNR and the channel stability $[6,7]$.

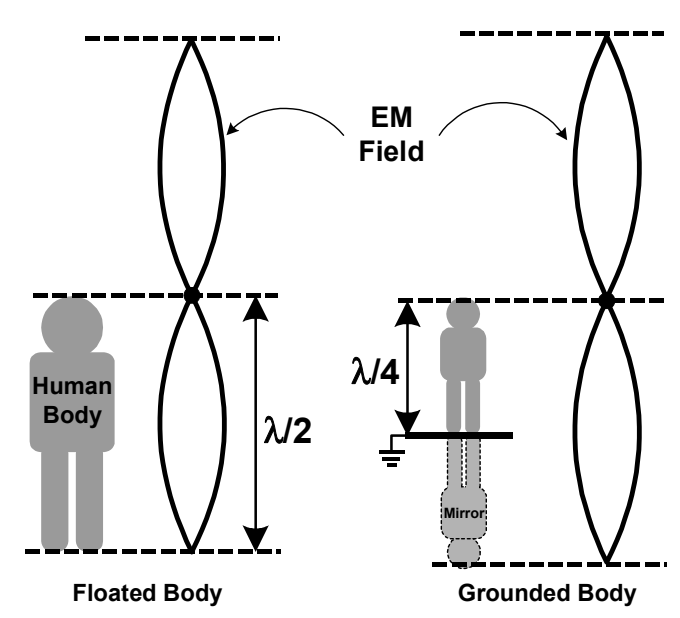

Figure 1. Human body antenna effect

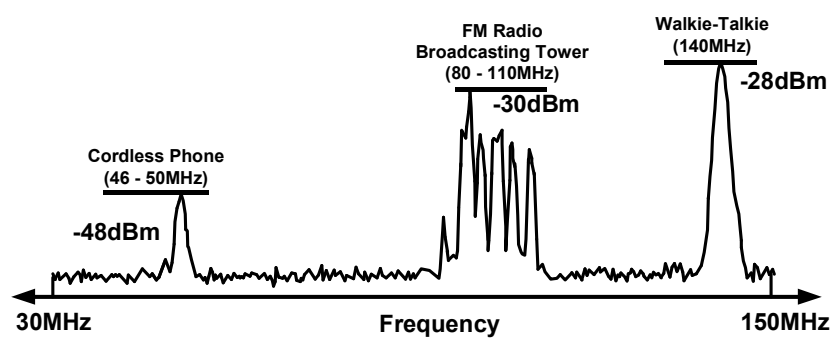

Figure 2. Measured in-band interferences to the body channel receiver 
The body antenna effect can inject various radio signals into a body channel receiver, and corrupt the desired signal severely. Fig. 2 shows the measured in-band interferences coupled to the receiver from wireless sources nearby. In order to measure the signal strength, a spectrum analyzer is connected to the right hand of the $1.8 \mathrm{~m}$ human subject using a metal electrode and a coaxial cable. Within the $1 \mathrm{~km}$ distance from the radio broadcasting tower, a number of FM signals exist on air, and their levels are up to $-38 \mathrm{dBm}$. When a walkietalkie is held by a BCC user, the power of the injected signal becomes even larger. For the secure $\mathrm{BCC}$, robustness to the SIR is more important than to the SNR.

\section{ADAPTIVE FREQUENCY HOPPING USING VARIABLE SUB-CHANNELS}

Unlike the random frequency hopping, the AFH monitors the status of the hopping channels continuously, and accepts only the clean channels for the communication. The AFH scheme was applied to the previous body channel transceiver for the purpose of interference rejection [4]. However, the transceiver is oriented to multimedia applications requiring high data rate. Therefore, the number of sub-channels is limited to 4 in the $30-120 \mathrm{MHz}$ frequency band, sacrificing the efficiency of the interference rejection. This work focuses on the communication reliability rather than the data rate for the healthcare applications. Fig. 3 shows the proposed AFH method using the variable number of sub-channels. Initially, the BCC performs the AFH with the 5 sub-channels, providing $2.5 \mathrm{Mb} / \mathrm{s}$ data rate. If lots of interfering signals exist around the transceiver and destroy most of the sub-channels, then the bandwidth of each sub-channel decreases, reducing the data rate. As the signal bandwidth reduces, the number of available sub-channels increases, and the channel utilization becomes better under lots of interferences. In addition, the narrower bandwidth improves the sensitivity of the receiver.

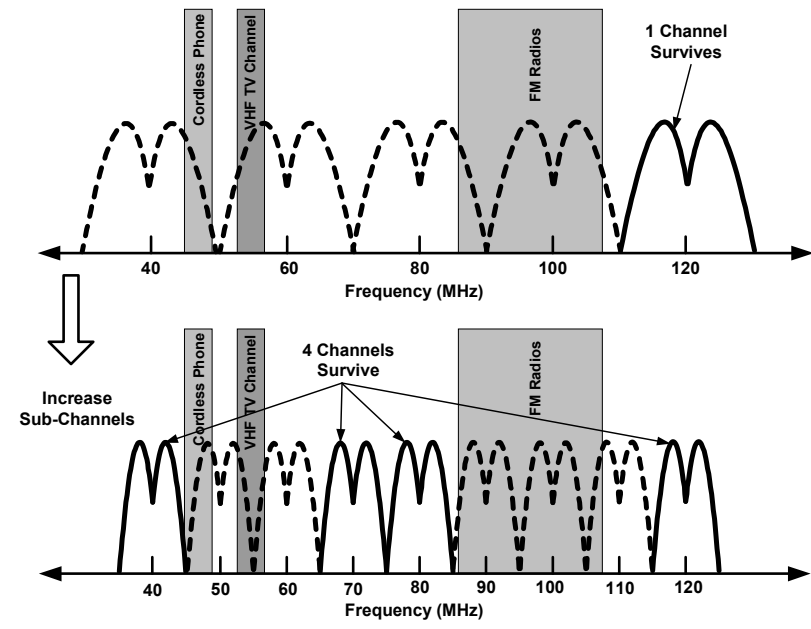

Figure 3. Adaptive frequency hopping using variable sub-channels

\section{TRANSCEIVER DEISGN}

\section{A. Transceiver Architecture}

Fig. 4 shows the architecture of the proposed AFH transceiver. The frequency-shift-keying (FSK) is adopted as the passband modulation. The FSK is suitable for the low power transceiver with low complexity since it has the constant envelope and enables the non-coherence demodulation. For the FSK, the control voltage of the VCO is directly modulated by the transmitted bit stream. The original data from the digital block is dc-free coded before the FSK modulator not to disturb the center frequency of the phase locked VCO. The variable bandwidth of the sub-channels is realized by the integer-N PLL. With the reference frequency of $F_{R E F}$, the output frequency ( $F_{\text {OUT }}$ ) of the PLL is represented as

$$
\mathrm{F}_{\mathrm{OUT}}=\frac{\mathrm{M} \bullet \mathrm{F}_{\mathrm{REF}}}{\mathrm{N}}
$$

where the division ratio $\mathrm{N}$ decides the channel bandwidth, and the multiplication factor $M$ decides which sub-channel is generated by the PLL. Using the $20 \mathrm{MHz}$ reference clock, the $\mathrm{N}$ and $\mathrm{M}$ values are programmed to give the scalable $100 \mathrm{~kb} / \mathrm{s}$ $-2.5 \mathrm{Mb} / \mathrm{s}$ data rate. The amplitude control unit following the DC-free encoder maintains the modulation index of the FSK signal to 2 by changing the control voltage swing of the VCO according to the data rate. Keeping the modulation index constant is necessary to make the signal spectrum confined to the variable bandwidth of the sub-channel. In the receiver chain, the direct conversion structure is chosen for the high integration level and the low power consumption. For the LO signal generation, the frequency synthesizer used in the FSK modulator is shared. After down conversion of the signal to zero-IF, a 4th order salen-key filter removes unwanted interferences injected to the receiver. To deal with the variable signal bandwidth, the resistors and the capacitors composing the low pass filter are made programmable. In contrast to the normal frequency hopping transceivers, the proposed architecture uses two frequency synthesizers for FSK modulation or LO generation. The advantage of the dual PLL structure is explained in the next section.

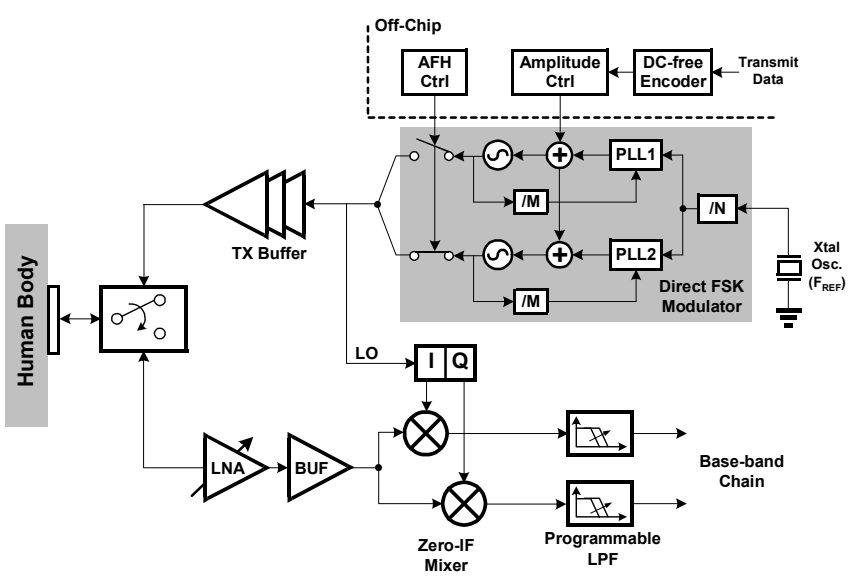

Figure 4. Archiecture of the proposed transceiver

\section{B. Frequency Hopping LO Generator with no Blank Time}

Because the minimum data rate of the proposed transceiver is $100 \mathrm{~kb} / \mathrm{s}$, the loop bandwidth of the frequency synthesizer should be less than $20 \mathrm{kHz}$ not to corrupt the signal spectrum of the transmitted bit stream. This results in the band 
switching time longer than $200 \mu$ s at the frequency hopping instant. Such a long response time of the PLL not only degrades the data throughput but also increases the acquisition time of the frequency hopping transceivers. The use of the dual frequency synthesizers can hide the long blank time completely, as shown in Fig. 5. When the PLL1 provides the required LO signal to the transceiver, the PLL2 changes its output frequency internally for the next hopping channel. At the moment of the frequency hopping, the PLL2 now provides the LO signal with the stabilized frequency, and the PLL1 starts to change its frequency. By performing this operating sequence continuously, the long band switching time of the PLL is not seen to the AFH transceiver. The power overhead due to the additional PLL can be controlled under $1 \mathrm{~mW}$ since its operating frequency is relatively low. Fig. 6 shows the internal structure of the low power PLL. The VCO which dissipates largest power in the PLL is implemented as a single-ended ring oscillator of which delay cell is current starved to reduce the current consumption by half. The source switching charge pump augmented with the fast-discharging switches M1, M2 enhances the current switching time significantly with only $10 \mathrm{uA}$ bias current. The retiming flipflop located in the feedback path removes jitter accumulated through the programmable divider. The FSK modulation is performed by varying the bias current of the delay cell according to the $\mathrm{V}_{\mathrm{MOD}}$.

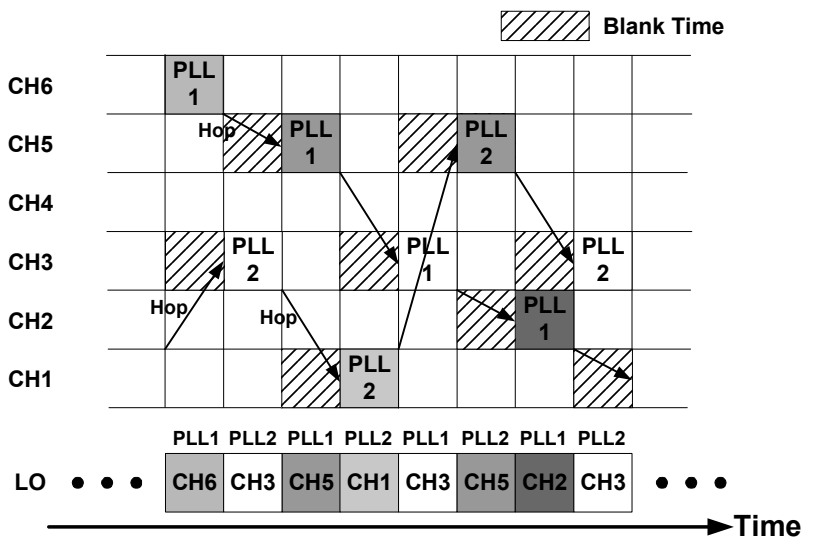

Figure 5. Frequency hopping with no band switching time

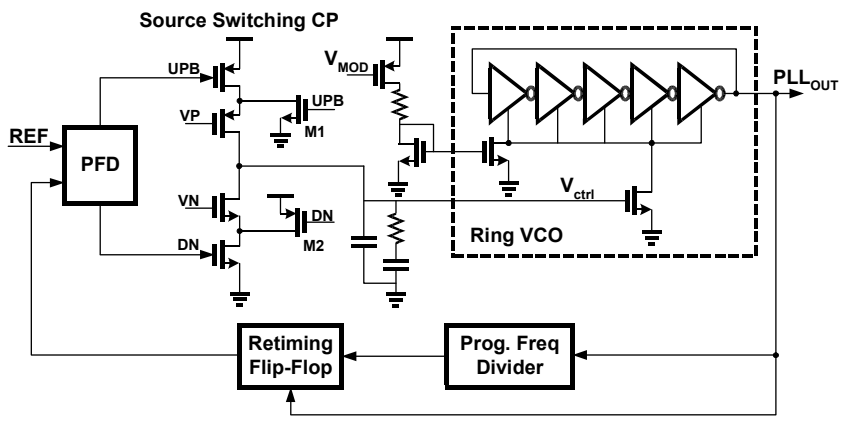

Figure 6. Low power Integer-N PLL

\section{Front-End Circuits}

Fig. 7 shows the front end circuits consisting of the LNA and the zero-IF mixer. The parallel combination of the commonsource (CS) and common-gate (CG) stages in the LNA converts the single-ended signal from the single electrode to the differential signal. Especially, the CG stage provides low impedance to the external LC band-select filter for its small size implementation and high quality factor. The amplifier having the diode-connected load drives the passive I/Q mixers with high linearity to prevent the desensitization of the desired signal by strong interferences. All of the transistors in the amplifier are PMOS devices, avoiding the nonlinearity due to the body effect.

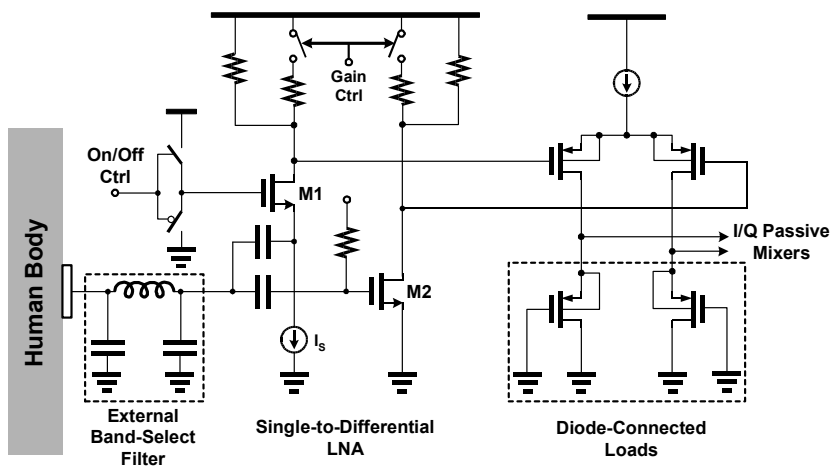

Figure 7. Cascade of the LNA and the mixer buffer

\section{IMPLEMENTATION RESULTS}

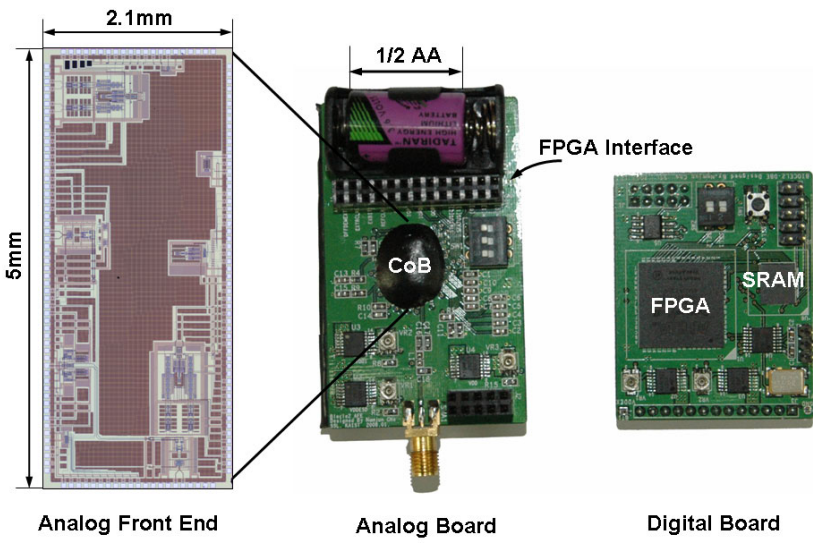

Figure 8. Test fixture

The analog part of the AFH transceiver is realized with $0.18 \mu \mathrm{m}$ CMOS process, and the digital blocks including the AFH controller and the DC-free encoder are implemented by FPGA. Fig. 8 depicts the test fixture evaluating the interference rejection of the proposed system. The TX board is operated by a half AA-size battery to eliminate its ground coupling with the RX board through the power line. The test board is connected to human skin with an $\mathrm{Ag} / \mathrm{AgCl}$ electrode. Fig. 9 shows the FSK signals modulated within the variable sub-channels. With the decreasing data rate, the bandwidth of the signal also decreases in proportion, because of the constant modulation index of 2. The time domain waveform of Fig. 10 confirms that the frequency hopping between sub-channels 
occurs with no blank time. Fig. 11 shows the gain curve of the receiver chain. The maximum gain of $36 \mathrm{~dB}$ is achieved at the center of the transfer curve, and the rejection of the adjacent channel is at least $43 \mathrm{~dB}$. When a sine tone is injected to the receiver as the interference, the SIR levels for $10^{-3} \mathrm{BER}$ are measured as $6 \mathrm{~dB}$ and $-25 \mathrm{~dB}$ at the in/out-band frequencies, respectively. Using the measured data from the analog front end, the throughput change of the proposed transceiver is simulated with the increasing number of interferences and displayed in Fig. 12. The signal-to-total interference power ratio is fixed to $-25 \mathrm{~dB}$ during the simulation. The $\mathrm{AFH}$ technique using the variable sub-channels apparently sustains $1.25 \mathrm{Mb} / \mathrm{s}$ throughput under the 10 interferences. Table $\mathrm{I}$ is the performance summary of the designed chip.

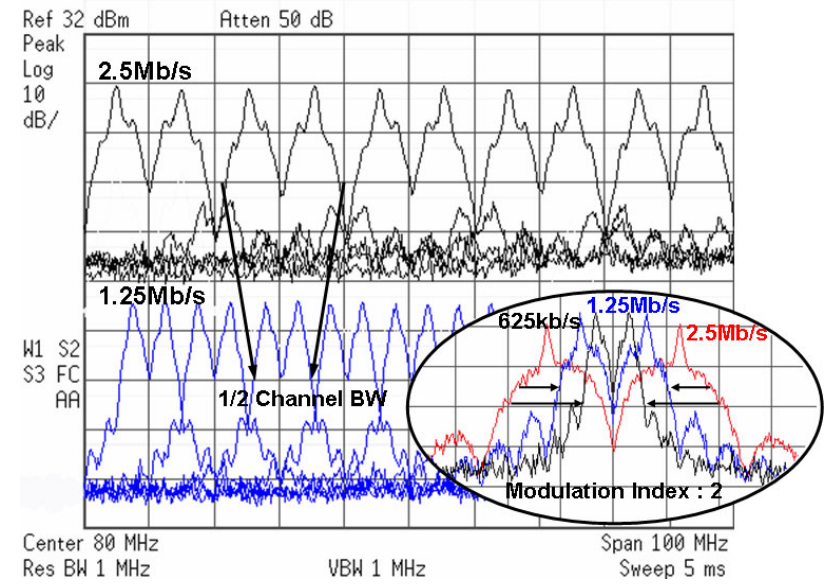

Figure 9. FSK spectrums in the variable sub-channels

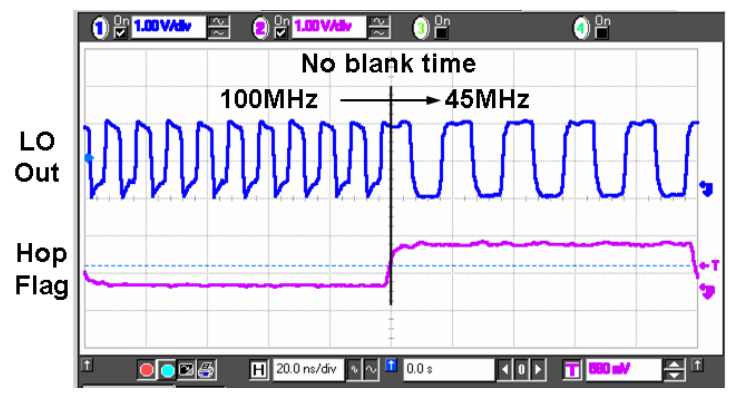

Figure 10. Frequency hopping with no blank time

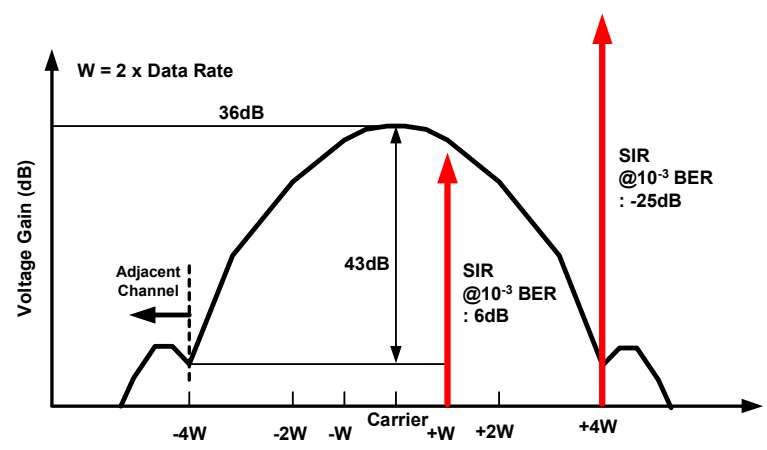

Figure 11. Voltage gain curve of the receiver chain

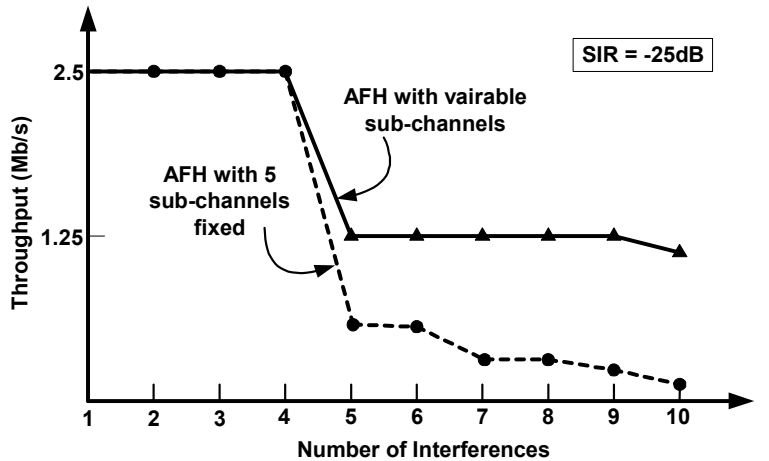

Figure 12. Data throughput under multiple interferences

TABLE I. PERFormance SUMmary

\begin{tabular}{|l|l|l|}
\hline Bandwidth & $30-120 \mathrm{MHz}$ with variable sub-channels \\
\hline Data Rate & $2.5 \mathrm{Mb} / \mathrm{s}-100 \mathrm{~kb} / \mathrm{s}$ scalable \\
\hline Sensitivity & $350 \mu \mathrm{V}-112 \mu \mathrm{V}$ scalable \\
\hline Voltage Gain & $36 \mathrm{~dB}$ \\
\hline SIR @ $10^{-3} \mathrm{BER}$ & $6 \mathrm{~dB}$ in-band, $-25 \mathrm{~dB}$ out-band \\
\hline \multirow{3}{*}{$\begin{array}{l}\text { Current } \\
\text { Consumption } \\
\text { @ } 1.5 \mathrm{~V} \text { supply }\end{array}$} & $\mathrm{TX}: 0.9 \mathrm{~mA}$ & RNA $: 0.6 \mathrm{~mA}$ \\
\cline { 2 - 3 } & PLL $: 0.8 \mathrm{~mA} \times 2$ & $\begin{array}{l}\text { Mixer + Filter: } 0.9 \mathrm{~mA} \\
\text { PLL }: 0.8 \mathrm{~mA} \times 2\end{array}$ \\
\cline { 2 - 3 } & $2.5 \mathrm{~mA}$ & $3.1 \mathrm{~mA}$ \\
\hline
\end{tabular}

\section{CONCLUSIONS}

The body-antenna effect degrades the reliability of the BCC severely by injecting a number of interferences to the receiver. To reject the body-induced signals efficiently, an adaptive frequency hopping technique changing the number of its sub-channels according to the communication environment is presented. Hiding the band-switching time with the dual frequency synthesizers eliminates the frequency hopping overhead of the proposed transceiver. The system designed with $0.18 \mu \mathrm{m}$ CMOS process can provide $1.25 \mathrm{Mb} / \mathrm{s}$ under $25 \mathrm{~dB}$ SIR caused by 10 interferences.

\section{REFERENCES}

[1] T. Handa, et al., "A very low-power consumption wireless ECG monitoring system using body as a signal transmission medium," Transducers '97, pp. $1003-1006$.

[2] M. Shinagawa, et al., "A near-field-sensing transceiver for intrabody communication based on the electrooptic effect," IEEE Trans. Instrum. Meas., vol. 53, pp. 1533 - 1538, Dec. 2004.

[3] T.G. Zimmerman, "Personal area networks: Near-field intrabody communication," IBM Syst. J., vol. 35, no. 3 - 4, pp. 609 - 617, 1996.

[4] N. Cho, et al, "A $60 \mathrm{~kb} / \mathrm{s}-$ to- $10 \mathrm{Mb} / \mathrm{s} \quad 0.37 \mathrm{~nJ} / \mathrm{b}$ adaptive-frequencyhopping transceiver for body-area network," in ISSCC Dig. Tech. Papers, Feb. 2008, pp. 132-133.

[5] P.J. Dimbylow, "FDTD calculations of the whole-body averaged SAR in an anatomically realistic voxel model of the human body from $1 \mathrm{MHz}$ to 1GHz," Phys. Medicine and Biology, vol. 42, pp. 479-490, 1997.

[6] K. Hachisuka, et al., "Development and performance analysis of an intra-body communicaion device," Transducers '03, pp. 1722 - 1725.

[7] N. Cho, et al, "The human body characteristics as a signal transmission medium for intrabody communication," IEEE Trans. Microw. Theory Tech., vol. 55, pp. 1080-1086, May. 2007. 